\title{
Full-zone valley polarization landscape of finite-momentum exciton in transition metal dichalcogenide monolayers
}

\author{
Ping-Yuan Lo $\odot,{ }^{*}$ Guan-Hao Peng, ${ }^{*}$ Wei-Hua Li, Yi Yang $\odot$, and Shun-Jen Cheng $\odot^{\dagger}$ \\ Department of Electrophysics, National Chiao Tung University, Hsinchu 30010, Taiwan \\ and Department of Electrophysics, National Yang Ming Chiao Tung University, Hsinchu 30010, Taiwan
}

(Received 9 October 2020; revised 16 November 2021; accepted 24 November 2021; published 20 December 2021)

\begin{abstract}
In this study we present a theoretical investigation of the full-zone landscape of finite-momentum dark excitons in $\mathrm{WSe}_{2}$-MLs by solving the density-functional-theory (DFT)-based Bethe-Salpeter equation (BSE) under the guidance of symmetry analysis. The studies reveal the comprehensive valley-polarization landscape of finitemomentum exciton of $\mathrm{WSe}_{2}$ monolayer. Dictated by the crystal symmetry, the valley pseudospin texture over the extended exciton-momentum $\boldsymbol{k}_{\text {ex }}$ space exhibits rich structures, featured by the inherently full valley polarizations in the excitonic $K_{\mathrm{ex}}, K_{\mathrm{ex}}^{\prime}$, and $Q_{\mathrm{ex}, i}$ valleys and also by the contrasted valley depolarizations for the exciton states lying in the $\overline{\Gamma_{\mathrm{ex}} M_{\mathrm{ex}, i}}$ paths. Attractively, the superior valley polarizations of the intervalley dark excitons in $\mathrm{WSe}_{2}-$ MLs are shown almost fully transferable to the optical polarization in the phonon-assisted photoluminescences because of the native suppression of exchange-induced depolarization in the second-order optical processes. The analysis of phonon-assisted photoluminescences accounts for the recently observed brightness, high degree of optical polarization, and long lifetime of the intervalley dark exciton states in tungsten-based TMD-MLs.
\end{abstract}

DOI: 10.1103/PhysRevResearch.3.043198

\section{INTRODUCTION}

Transition metal dichalcogenide monolayers (TMD-MLs) have drawn a broad interest in recent years because of the intriguing spin-valley-coupled characteristics in the electronic and excitonic structures [1-6]. As a massive Dirac material, the band structure of a TMD-ML is characterized by the interband energy gaps opened in the distinct valleys located at the $K$ and $K^{\prime}$ corners of the first Brillouin zone (BZ) that follow the opposite optical helicity and allow for the valleyselective optical excitation and manipulation [4-6]. With the extraordinary valley-excitonic properties, TMD-MLs serve as promising nanomaterials for the application of valley-based photonics as long as the degree of valley polarization of exciton can remain high and well transferrable to the optical polarization of the emitted photons $[7,8]$.

However, it is known that the valley polarization of a bright exciton in a TMD-ML is very likely depolarized by the intrinsic electron-hole $(e-h)$ exchange interaction that couples the interband excitations in the distinct valleys [4,913]. Despite the weak meV-scale coupling strength, the momentum-conserving $e-h$ exchange interaction (EHEI) can efficiently intermix two exchange-free excitations in the $K$ and $K^{\prime}$ valleys that hold the same momentum and similar energy

\footnotetext{
*These authors contributed equally to this work and their names are listed by alphabetical order.

†sjcheng@mail.nctu.edu.tw

Published by the American Physical Society under the terms of the Creative Commons Attribution 4.0 International license. Further distribution of this work must maintain attribution to the author(s) and the published article's title, journal citation, and DOI.
}

(quasidegenerate) $[4,14,15]$. Note that, at the most general level, the fundamental time-reversal symmetry (TRS) ensures the degeneracy only for the excitations in the opposite valleys that carry the opposite momentum. Hence, even without any spatial symmetries, the distinct degenerate valley-exciton states that hold the same nearly vanishing momentum, i.e., the bright exciton (BX) states, meet the both criteria and natively suffer from the exchange-induced valley depolarization. By contrast, the valley exciton states with the same finite momenta, i.e., momentum-forbidden dark exciton (MFDX) states, are not enforced by the TRS to be degenerate and, unlike the BXs, their valley polarizations are purely dictated by the crystal symmetries and should be momentum dependent.

In spite of violating the momentum selection rules, those MFDXs in TMD-MLs have drawn massive attention recently because of their essential involvement in various optical and dynamics phenomena [15-29]. Very recently, direct generation and probe of finite-momentum excitons in $\mathrm{WSe}_{2}$-MLs have been realized by using the integrated technology of optical pump-probe and angle-resolved photoemission spectroscopies [28,29]. Moreover, the latest cryogenic photoluminescence (PL) measurements on tungsten-based TMD-MLs have revealed the pronounced optical signatures of the intervalley MFDXs which are significantly bright [17], highly polarized [16,18], and long lived [30]. Such long-lived and optically accessible MFDXs are attractive for the quantum applications and realizing excitonic Bose-Einstein condensation (BEC) [31-34]. With the recently achieved experimental advances in the investigation of MFDX, establishing a comprehensive theoretical understanding of those MFDXs in TMD-MLs over the extended momentum space is highly demanded, which yet remains rarely explored so far [35].

In this paper we present a comprehensive theoretical investigation of the full-zone band structures and valley 
polarizations of finite-momentum exciton states of $\mathrm{WSe}_{2}$ MLs by numerically solving the DFT-based BSE under the guidance of symmetry analysis [36]. The studies reveal the symmetry-dictated nature of the valley and optical properties of the finite-momentum exciton states. It is shown that the activeness of electron-hole exchange, being the main cause of valley depolarization, of exciton is highly momentum dependent under the enforcement of crystal symmetry. It turns out that the valley polarization of the finite-momentum exciton exhibits a rich skyrmionlike valley pseudospin texture over the extended momentum space, contrasted by the full valley polarizations for the intervalley MFDX states in the excitonic $K_{\mathrm{ex}}, K_{\mathrm{ex}}^{\prime}$, and $Q_{\mathrm{ex}, i}$ valleys and the suppressed valley polarizations for the exciton states lying in the $\overline{\Gamma_{\mathrm{ex}} M_{\mathrm{ex}}}$ path as well. In the second-order perturbation theory, we show that the inherently high valley polarization of the intervalley dark exciton is almost fully transferable to the optical polarizations of indirect phonon-assisted luminescences. The theoretical analysis accounts for the recently observed spectral brightness [16-18], high degree of optical polarization [16,18], and long-lived dynamics of the intervalley MFDXs in tungstenbased TMD-MLs [30].

\section{THEORETICAL ANALYSIS AND NUMERICAL METHODOLOGY}

\section{A. Exciton theory}

First, we consider the exciton state with the center-of-mass wave vector $\boldsymbol{k}_{\mathrm{ex}}, \quad\left|S, \boldsymbol{k}_{\mathrm{ex}}\right\rangle=$ $\frac{1}{\sqrt{\mathcal{A}}} \sum_{v c \boldsymbol{k}} A_{S, \boldsymbol{k}_{\mathrm{e}}}(v c \boldsymbol{k}) \hat{c}_{c, \boldsymbol{k}+\boldsymbol{k}_{\mathrm{ex}}^{\dagger}} \hat{h}_{v,-\boldsymbol{k}}^{\dagger}|G S\rangle$, written as a linear combination of the configurations of the electron-hole $(e-h)$ pairs, $\hat{c}_{c, \boldsymbol{k}+\boldsymbol{k}_{\mathrm{ex}}}^{\dagger} \hat{h}_{v,-\boldsymbol{k}}^{\dagger}|G S\rangle$, where the particle operator $\hat{c}_{c, \boldsymbol{k}}^{\dagger}$ $\left(\hat{h}_{v,-k}^{\dagger}\right)$ is defined to create the electron (hole) of the wave vector $\boldsymbol{k}(-\boldsymbol{k})$ in the conduction band $c$ (valence band $v$ ) from the ground state of the system with the fully filled valence bands $|G S\rangle, S$ is the index of exciton band, $A_{S, \boldsymbol{k}_{\mathrm{e}}}(v c \boldsymbol{k})$ is the amplitude of the $e-h$ configuration $\hat{c}_{c, k+k_{\text {ex }}}^{\dagger} \hat{h}_{v,-k}^{\dagger}|G S\rangle$, and $\mathcal{A}$ is the area of the two-dimensional (2D) material. Note that the spin states of the conduction and valence bands and those of the exciton states as well are implicitly absorbed in their band indices, $c, v$, and $S$, respectively. The exciton wave function in the reciprocal $\boldsymbol{k}$ space, $A_{S, \boldsymbol{k}_{\mathrm{e}}}(v c \boldsymbol{k})$, follows the BSE that reads [35-42]

$$
\begin{aligned}
& {\left[\epsilon_{c, \boldsymbol{k}+\boldsymbol{k}_{\mathrm{ex}}}-\epsilon_{v, \boldsymbol{k}}-E_{S, \boldsymbol{k}_{\mathrm{ex}}}^{X}\right] A_{S, \boldsymbol{k}_{\mathrm{ex}}}(v c \boldsymbol{k})} \\
& \quad+\sum_{v^{\prime} c^{\prime} \boldsymbol{k}^{\prime}} U_{\boldsymbol{k}_{\mathrm{ex}}}\left(v c \boldsymbol{k}, v^{\prime} c^{\prime} \boldsymbol{k}^{\prime}\right) A_{S, \boldsymbol{k}_{\mathrm{ex}}}\left(v^{\prime} c^{\prime} \boldsymbol{k}^{\prime}\right)=0,
\end{aligned}
$$

where $E_{S, \boldsymbol{k}_{\mathrm{e}}}^{X}$ is the eigenenergy of the exciton state $\left|S, \boldsymbol{k}_{\mathrm{ex}}\right\rangle$, $\epsilon_{c, \boldsymbol{k}+\boldsymbol{k}_{\mathrm{ex}}}\left(-\epsilon_{v, \boldsymbol{k}}\right)$ is the kinetic energy of free electron (hole) in the conduction (valence) band, and $U_{\boldsymbol{k}_{\mathrm{ex}}}\left(v c \boldsymbol{k}, v^{\prime} c^{\prime} \boldsymbol{k}^{\prime}\right)$ is the kernel of $e$ - $h$ Coulomb interaction consisting of the screened $e-h$ direct interaction and the $e-h$ exchange one, $U_{\boldsymbol{k}_{\mathrm{ex}}}=-V_{\boldsymbol{k}_{\mathrm{ex}}}^{d}+$ $V_{\boldsymbol{k}_{\mathrm{ex}}}^{x}$. The explicit definitions of the matrix elements of $V_{\boldsymbol{k}_{\text {ex }}}^{d}$ and $V_{\boldsymbol{k}_{\mathrm{ex}}}^{x}$ in terms of the Bloch wave functions and dielectric function are given in Ref. [43]. Throughout this work, the screening in the direct $e-h$ Coulomb interaction is modeled by using the Keldysh formalism [40,43-49].
The quasiparticle band structures of $\mathrm{WSe}_{2}-\mathrm{MLs}$ are calculated using the first-principles VASP package [50] within the Heyd-Scuseria-Ernzerhof (HSE) functional model [51], as presented in Fig. 1(b) and Fig. S1 in Ref. [43]. Figures 1(c) and 1(d) show the energy contour of the lowest conduction band of a WSe $-\mathrm{ML}$ over the first $\mathrm{BZ}$ in the electron-momentum $\left[\boldsymbol{k}=\left(k_{x}, k_{y}\right)\right]$ space, with the indication of the high symmetry points $\Gamma, K / K^{\prime}, M_{i}$ and $Q_{i} / Q_{i}^{\prime}$.

Figure 1(e) shows the calculated lowest exciton band structure of a $\mathrm{WSe}_{2}-\mathrm{ML}$ over the $\mathrm{BZ}$ in the exciton-momentum $\left[\boldsymbol{k}_{\mathrm{ex}}=\left(k_{\mathrm{ex}, x}, k_{\mathrm{ex}, y}\right)\right]$ space. The full-zone calculations of the exciton band structures of TMD-MLs are achieved by using our newly developed methodology [36], allowing for efficiently solving the BSE established in the Wannier tight-binding (TB) scheme [52-54] on the first-principles base $[43,55,56]$.

It is known that the direct $e-h$ Coulomb interaction makes the predominant contribution to the large binding energy of exciton in a TMD-ML, but no direct effect on the intervalley couplings for exciton $[57,58]$. By contrast, the momentumconserving EHEI could couple the exciton states in distinct valleys that carry the same exciton momentum $\boldsymbol{k}_{\mathrm{ex}}$ and leads to the unwanted valley depolarization of exciton in the valley-based applications $[9,10]$. As previously stated, the $e-h$ exchange interaction is especially efficient to couple the distinct valley excitons with the same momentum and similar energy (quasidegeneracy). Since the degeneracies of MFDX states are dictated by the crystal symmetries, so are the valley polarizations of the exciton states.

\section{B. Symmetry analysis}

Thus, we conduct the symmetry analysis below to predict the degeneracies of the exchange-free exciton states $\left|S, \boldsymbol{k}_{\mathrm{ex}}\right\rangle^{(0)}$ of TMD-MLs under the $D_{3 h}$-group symmetry [59]. Throughout this work, the superscript (0) indicates the absence of electron-hole exchange interaction. Consider two distinct free $e-h$ pair states with the same $\boldsymbol{k}_{\mathrm{ex}}$ excited from the different valence states at $\boldsymbol{k}$ and $\boldsymbol{k}^{\prime}$. They are degenerate as $\epsilon_{c, \boldsymbol{k}+\boldsymbol{k}_{\mathrm{ex}}}-\epsilon_{v, \boldsymbol{k}}=\epsilon_{c, \boldsymbol{k}^{\prime}+\boldsymbol{k}_{\mathrm{ex}}}-\epsilon_{v, \boldsymbol{k}^{\prime}}$, which can generally hold only if $\epsilon_{v, \boldsymbol{k}}=\epsilon_{v, \boldsymbol{k}^{\prime}}$ and $\epsilon_{c, \boldsymbol{k}+\boldsymbol{k}_{\mathrm{ex}}}=\epsilon_{c, \boldsymbol{k}^{\prime}+\boldsymbol{k}_{\mathrm{ex}}}$. From the theory of group representations, the above two equations hold when the space group symmetry of the TMD-ML satisfies both equations $\boldsymbol{k}^{\prime}=\hat{U} \boldsymbol{k}$ and $\boldsymbol{k}^{\prime}+$ $\boldsymbol{k}_{\mathrm{ex}}=\hat{U}\left(\boldsymbol{k}+\boldsymbol{k}_{\mathrm{ex}}\right)$, for any symmetry operator $\hat{U} \in D_{3 h}=$ $\left\{\hat{E}, \hat{C}_{3}, \hat{C}_{3}^{-1}, \hat{\sigma}_{h}, \hat{S}_{3}, \hat{S}_{3}^{-1}, \hat{C}_{2,1}^{\prime}, \hat{C}_{2,2}^{\prime}, \hat{C}_{2,3}^{\prime}, \hat{\sigma}_{v, 1}, \hat{\sigma}_{v, 2}, \hat{\sigma}_{v, 3}\right\}$. Accordingly, we find the criterion for the formation of valley degeneracy of two distinct $e-h$ pairs carrying the same $\boldsymbol{k}_{\mathrm{ex}}$, i.e.,

$$
\boldsymbol{k}_{\mathrm{ex}}=\hat{U} \boldsymbol{k}_{\mathrm{ex}}
$$

Applying all the symmetry operators $\hat{U} \in D_{3 h}$ in Eq. (2) for all $\boldsymbol{k}_{\mathrm{ex}} \in \mathrm{BZ}$ [43], one can show that distinct $e$ - $h$ pair states with the common $\boldsymbol{k}_{\mathrm{ex}}$ could be valley degenerate only if $\boldsymbol{k}_{\mathrm{ex}}$ lies along the lines connecting the $\Gamma_{\mathrm{ex}}$ and $M_{\mathrm{ex}, i}$ points, i.e., the axes associated with the $3 \hat{\sigma}_{v}$ and $3 \hat{C}_{2}^{\prime}$ symmetries, as shown in Fig. 1(a). The matrix representations of $\hat{U} \in D_{3 h}$ in the Cartesian coordinates are given in Ref. [43]. This predicts the exchange-induced valley depolarization that impacts only 
(a)

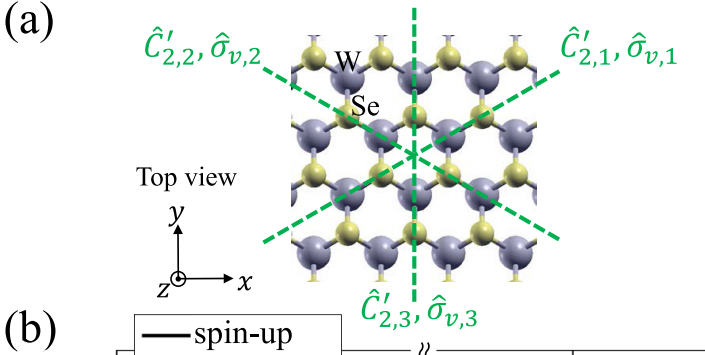

(b)

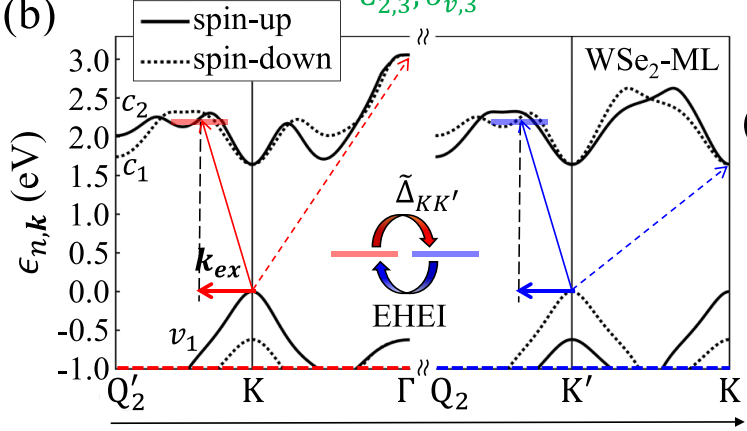

(c)

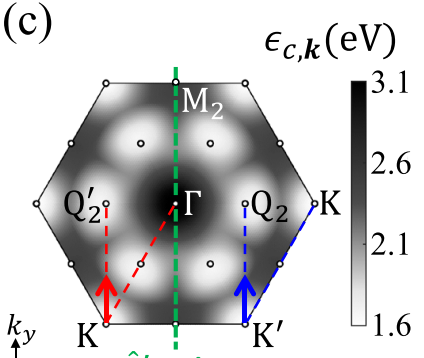

(e)
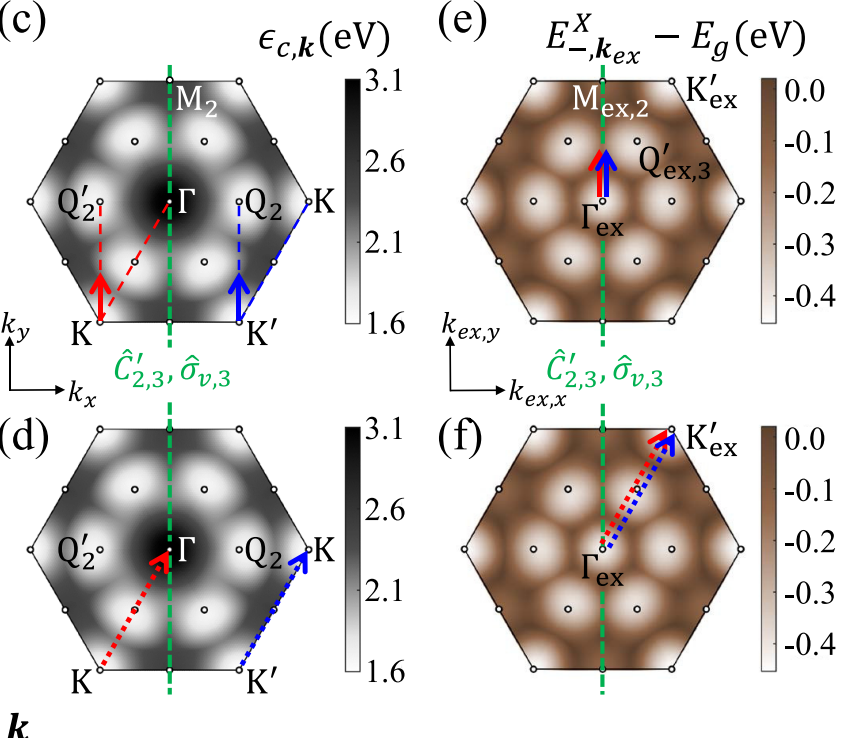

(f)

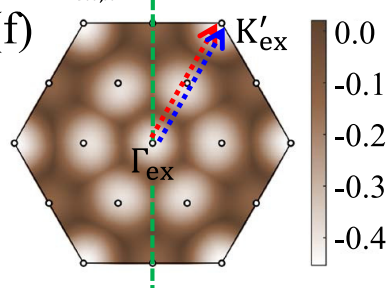

FIG. 1. (a) Top view of the lattice structure of a TMD-ML with $D_{3 h}$ symmetry. The green dashed lines indicate the three axes with respect to the $C_{2}^{\prime}$ rotational and $\sigma_{v}$ mirror symmetries. (b) The DFT-calculated quasiparticle conduction and valence bands of a WSe ${ }_{2}$-ML along the $\boldsymbol{k}$ paths indicated by the red and blue dashed lines in (c). (c) and (d) Energy contour plot of the DFT-calculated lowest conduction band of a $\mathrm{WSe}_{2}$-ML over the first BZ in the electron-momentum (k) space. (e) and (f) Energy contour plot of the lowest exciton band of a WSe $\mathrm{HL}_{2} \mathrm{ML}$ over the first BZ in the exciton-momentum $\left(\boldsymbol{k}_{\mathrm{ex}}\right)$ space. The red (blue) arrow lines in both (c) and (e) indicate the $e$ - $h$ pair excitation from the valence $K\left(K^{\prime}\right)$ valley to a conduction state at the $\overline{K Q_{2}^{\prime}}\left(\overline{K^{\prime} Q_{2}}\right)$ path, carrying the exciton momentum $\boldsymbol{k}_{\mathrm{ex}} \| \overline{\Gamma_{\mathrm{ex}} M_{\mathrm{ex}, 2}}$. The dashed red and blue arrow lines in (d) and (f) indicate the two distinct valley excitations carrying the same $\boldsymbol{k}_{\mathrm{ex}} \| \overline{\Gamma_{\mathrm{ex}} K_{\mathrm{ex}}^{\prime}}$. In the view of the $\hat{\sigma}_{v, 3}$ symmetry, one can realize that the two distinct valley excitations in (c) carrying the same $\boldsymbol{k}_{\mathrm{ex}}$ are degenerate, while the two ones in (d) are not.

the exciton states with the specific $\boldsymbol{k}_{\mathrm{ex}} \in \overline{\Gamma_{\mathrm{ex}} M_{\mathrm{ex}, i}}$, including the commonly known BX states around the $\Gamma_{\text {ex }}$ point $[9,10]$.

As an illustrative instance, Fig. 1(c) exemplifies the two distinctive $e$ - $h$ pair excitations with the same $\boldsymbol{k}_{\mathrm{ex}}$ along the $k_{\mathrm{ex}, y}$ direction, i.e., $\overline{\Gamma_{\mathrm{ex}} M_{\mathrm{ex}, 2}}$, which are excited from the valence $K$ towards the conduction $Q_{2}^{\prime}$ valley (red arrow line) and from the valence $K^{\prime}$ towards the conduction $Q_{2}$ valley (blue arrow line), respectively. In Fig. 1(b) one can identify the transition energies of the two free valley excitations to be the same. For comparative illustration, we consider another set of two valley transitions excited with the common $\boldsymbol{k}_{\mathrm{ex}}$ along $\overline{\Gamma_{\mathrm{ex}} K_{\mathrm{ex}}^{\prime}}$, as indicated by the dashed arrow lines in Fig. 1(d). With the misaligned $\boldsymbol{k}_{\mathrm{ex}}$ from $\overline{\Gamma_{\mathrm{ex}} M_{\mathrm{ex}, i}}$ the $K \Gamma$ and $K^{\prime} K$ transition energies are apparently different as predicted and shown in Fig. 1(b).

Beyond the noninteracting $e-h$ pair states, the above symmetry analysis remains valid for the exchange-free exciton states. Figure 2(a) shows the calculated energy band dispersions of exchange-free exciton $E_{S, \boldsymbol{k}_{\text {ex }}}^{X(0)}$ of a WSe $\mathrm{WL}_{2}$-Mith the $\boldsymbol{k}_{\mathrm{ex}}$ along $\overline{\Gamma_{\mathrm{ex}} M_{\mathrm{ex}, 2}}$ and $\overline{\Gamma_{\mathrm{ex}} K_{\mathrm{ex}}^{\prime}}$ directions, solved from the exchange-free BSE including the direct part of Coulomb interaction only. In the absence of EHEI, the energy bands of the lowest exciton doublet along $\overline{\Gamma_{\mathrm{ex}} M_{\mathrm{ex}, 2}}$ does remain degenerate while the ones along $\overline{\Gamma_{\mathrm{ex}} K_{\mathrm{ex}}^{\prime}}$ are shown valley split. Figure 2(b) presents the energy splitting of the lowest exchange-free spinlike exciton doublet $\Delta_{+-}^{(0)}\left(\boldsymbol{k}_{\mathrm{ex}}\right) \equiv E_{+, \boldsymbol{k}_{\mathrm{ex}}}^{X(0)}-E_{-, \boldsymbol{k}_{\mathrm{ex}}}^{X(0)}$ (where the subscript $+/-$ indicates the upper/lower band, and the superscript (0) indicates the absence of EHEI), as a function of $\boldsymbol{k}_{\mathrm{ex}}$ over the BZ, indeed showing $\Delta_{+-}^{(0)}\left(\boldsymbol{k}_{\mathrm{ex}}\right)=0$ (magenta lines) along the three $\overline{\Gamma_{\mathrm{ex}} M_{\mathrm{ex}, i}}$ paths. Besides the vanishing energy splittings at $\overline{\Gamma_{\mathrm{ex}} M_{\mathrm{ex}, i}}$ axes, one also find the degeneracies of the exchange-free states lying between the $K_{\mathrm{ex}}$ and $Q_{\mathrm{ex}}$ valleys, which belong to the accidental degeneracies.

\section{RESULTS AND DISCUSSION}

\section{A. Full-zone band structures and valley polarizations of finite-momentum exciton}

Figure 2(d) shows the calculated exciton bands of the $\mathrm{WSe}_{2}-\mathrm{ML}$ with the full consideration of the both $e-h$ direct and exchange interactions. In the view of dipole-allowed spinlike exciton states of $\mathrm{WSe}_{2}-\mathrm{ML}$, the calculated $K^{\prime} K$ MFDX states in the $K_{\mathrm{ex}}^{\prime}$ valley, $|D\rangle \equiv\left|-, \boldsymbol{K}_{\mathrm{ex}}^{\prime}\right\rangle$, is energetically lower than the $\mathrm{BX}$ states in the radiative light cone (LC), $|B\rangle \equiv$ $\left| \pm, \boldsymbol{k}_{\mathrm{ex}} \in \mathrm{LC}\right\rangle$, by $\Delta E_{B D}^{X} \approx 29 \mathrm{meV}$, in agreement with the measured value of $32 \mathrm{meV}$ by Ref. [18]. Under the effect of EHEI, the BX states around the $\Gamma_{\text {ex }}$ as well as the MFDX ones along the $\overline{\Gamma_{\mathrm{ex}} M_{\mathrm{ex}, i}}$ paths no longer remain degenerate and, as shown in Figs. 2(d) and 2(e), become valley split with $\Delta_{+-}\left(\boldsymbol{k}_{\mathrm{ex}}\right) \equiv E_{+, \boldsymbol{k}_{\mathrm{ex}}}^{X}-E_{-, \boldsymbol{k}_{\mathrm{ex}}}^{X} \approx 2\left|\tilde{\Delta}_{K K^{\prime}}\left(\boldsymbol{k}_{\mathrm{ex}}\right)\right| \neq 0$, where $\tilde{\Delta}_{K K^{\prime}}\left(\boldsymbol{k}_{\mathrm{ex}}\right)$ represents the $e$ - $h$ exchange interaction that intermixes the $K$ and $K^{\prime}$ valley components in the exciton states with $\boldsymbol{k}_{\mathrm{ex}}$. Throughout this work, the momentum-dependent EHEIs, $\tilde{\Delta}_{K K^{\prime}}\left(\boldsymbol{k}_{\mathrm{ex}}\right)$, in the MFDX states are fully taken into account in both numerical calculations and analysis. For a valley-polarized exciton state $\left|S, \boldsymbol{k}_{\mathrm{ex}}\right\rangle=\alpha_{S, \boldsymbol{k}_{\mathrm{ex}}}^{K}\left|K, \boldsymbol{k}_{\mathrm{ex}}\right\rangle^{(0)}+$ $\alpha_{S, \boldsymbol{k}_{\mathrm{ex}}}^{K^{\prime}}\left|K^{\prime}, \boldsymbol{k}_{\mathrm{ex}}\right\rangle^{(0)}$, intermixed with the $K$ and $K^{\prime}$ valley components, the degree of valley polarization (DoV) of the exciton is quantified by $P_{S, \boldsymbol{k}_{\mathrm{ex}}}^{v} \equiv \frac{\left|\alpha_{S, \mathrm{k}_{\mathrm{ex}}}^{K}\right|^{2}-\left|\alpha_{S, \mathrm{k}_{\mathrm{ex}}}^{K^{\prime}}\right|^{2}}{\left|\alpha_{S, \mathrm{k}_{\mathrm{ex}}}^{K}\right|^{2}+\left|\alpha_{S, \boldsymbol{k}_{\mathrm{ex}}}^{K_{\mathrm{e}}}\right|^{2}}$. As seen in Fig. 2(f), 
(a)

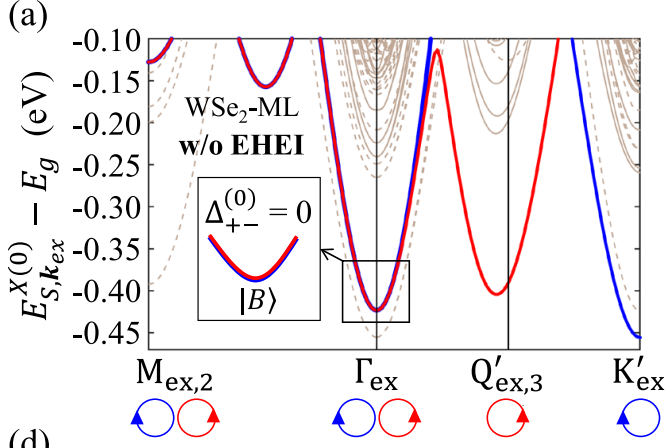

(d)

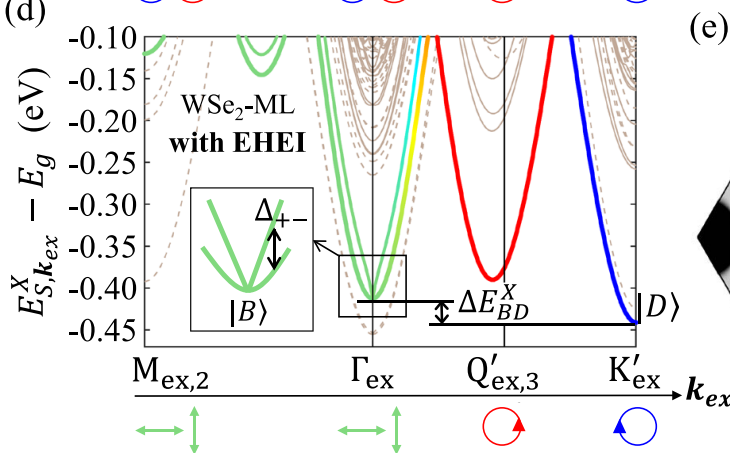

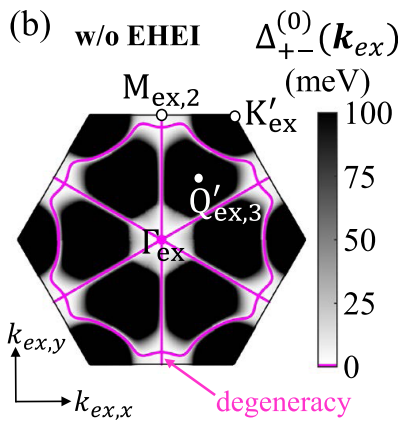

(e) with EHEI

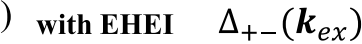

(c)

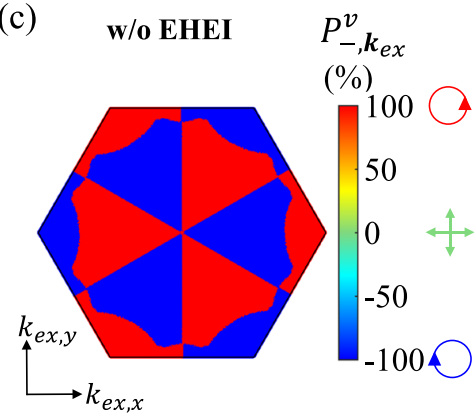

(f)

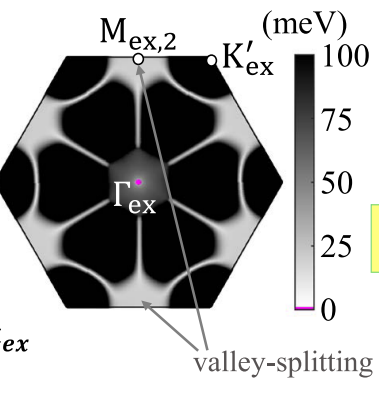

(1)

(1)
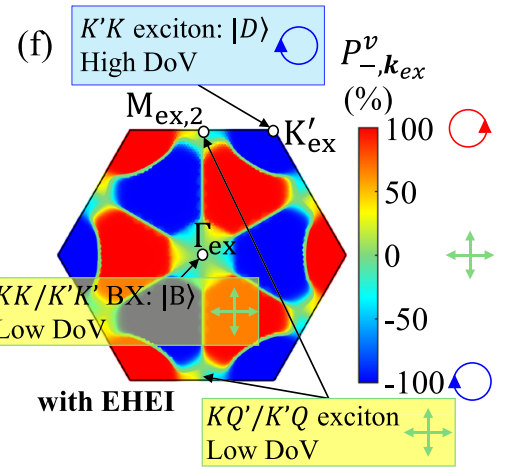

FIG. 2. (a) Exciton band structure of a WSe ${ }_{2}$-ML along the paths of $\overline{\Gamma_{\mathrm{ex}} M_{\mathrm{ex}, 2}}$ and $\overline{\Gamma_{\mathrm{ex}} K_{\mathrm{ex}}^{\prime}}$ calculated with the neglect of the $e-h$ exchange interaction (EHEI). Solid (dashed) lines: Spinlike (-unlike) exciton bands. The band colors follow the color-bar scales of (c) and (f) to represent the valley degree of polarization of the lowest exciton band. (b) The $\boldsymbol{k}_{\mathrm{ex}}$-dependent energy differences $\Delta_{+-}^{(0)}\left(\boldsymbol{k}_{\mathrm{ex}}\right) \equiv E_{+, \boldsymbol{k}_{\mathrm{ex}}}^{X(0)}-E_{-, \boldsymbol{k}_{\mathrm{ex}}}^{X(0)}$ between the lowest exchange-free spinlike exciton doublet $\left| \pm, \boldsymbol{k}_{\mathrm{ex}}\right\rangle^{(0)}$, based on the result of (a). The magenta-colored line indicates $\Delta_{+-}^{(0)}\left(\boldsymbol{k}_{\mathrm{ex}}\right)=0$ (the degeneracy of the exciton states). (c) The $\boldsymbol{k}_{\text {ex }}$-dependent valley polarization $P_{-, \boldsymbol{k}_{\mathrm{ex}}}^{v}$ of the lowest spinlike exciton states $\left|-, \boldsymbol{k}_{\mathrm{ex}}\right\rangle$, based on (a). Under no effect of EHEI, all the exciton states in the BZ are shown fully $K$ - or $K^{\prime}$-valley polarized. (d)-(f) The same as (a)-(c) but with the inclusion of the $e-h$ exchange interaction in the calculation. $\Delta E_{B D}^{X}=29 \mathrm{meV}$ for $\mathrm{WSe}_{2}-\mathrm{ML}$. The inset of (d) shows the exchange-induced valley-splitting $\Delta_{+-}\left(\boldsymbol{k}_{\text {ex }}\right)$ of the BX doublet at $\boldsymbol{k}_{\text {ex }}$ in the small light cone. Comparing (a)-(c) with (d)-(f), one notes that the EHEI makes the

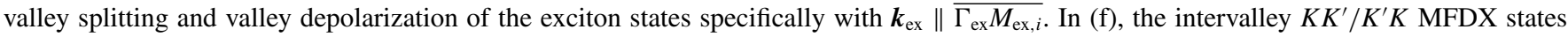
around the $K_{\mathrm{ex}} / K_{\mathrm{ex}}^{\prime}$ valleys remarkably remain fully valley polarized even under the action of EHEI.

the exciton states lying on the $\overline{\Gamma_{\mathrm{ex}} M_{\mathrm{ex}, i}}$ paths under the impact of EHEI are featured with $P_{-, \boldsymbol{k}_{\mathrm{ex}}}^{v} \sim 0$, while the MFDX ones apart from the $\overline{\Gamma_{\mathrm{ex}} M_{\mathrm{ex}, i}}$ paths, like those around $Q_{\mathrm{ex}, i} / Q_{\mathrm{ex}, i}^{\prime}$ and $K_{\text {ex }} / K_{\text {ex }}^{\prime}$ valleys, retain the high DoV of $\left|P_{-, \boldsymbol{k}_{\text {ex }}}^{v}\right| \lesssim 100 \%$.

For more illustration of the momentum-dependent valley polarization of exciton, we take the lowest exchange-free exciton doublet with the well-defined valley component $(K$ or $\left.K^{\prime}\right)$ as basis, $\left|S=K, \boldsymbol{k}_{\mathrm{ex}}\right\rangle^{(0)}$ and $\left|S=K^{\prime}, \boldsymbol{k}_{\mathrm{ex}}\right\rangle^{(0)}$, to construct the pseudospin model for the lowest valley-polarized exciton states $[2,3,5]$. In the pseudospin model, the effective Hamiltonian of valley exciton doublet can be written as a $2 \times 2$ matrix and formulated as $\hat{H}_{X}=\boldsymbol{\Omega}_{X} \cdot \hat{\boldsymbol{\sigma}}$, in terms of the spin-1/2 Pauli matrices, $\hat{\boldsymbol{\sigma}}=\sigma_{x} \hat{\boldsymbol{x}}+\sigma_{y} \hat{\boldsymbol{y}}+\sigma_{z} \hat{z}$ and the $\boldsymbol{k}_{\mathrm{ex}}$-dependent effective field $\boldsymbol{\Omega}_{X}=\boldsymbol{\Omega}_{X, x} \hat{\boldsymbol{x}}+\boldsymbol{\Omega}_{X, y} \hat{\boldsymbol{y}}+\boldsymbol{\Omega}_{X, z} \hat{z}$ which is determined on the first-principles base in this work (see Ref. [43] for details). For brevity we omit the explicit indication of $\boldsymbol{k}_{\mathrm{ex}}$ in the formalisms here. Expressing the pseudomagnetic field as $\boldsymbol{\Omega}_{X}=\left|\boldsymbol{\Omega}_{X}\right|(\sin \theta \cos \phi \hat{\boldsymbol{x}}+\sin \theta \sin \phi \hat{\boldsymbol{y}}+\cos \theta \hat{\boldsymbol{z}})$, the lower exciton state $(S=-)$ is solved as

$$
\begin{aligned}
|-\rangle & =\alpha_{-}^{K}|K\rangle^{(0)}+\alpha_{-}^{K^{\prime}}\left|K^{\prime}\right\rangle^{(0)} \\
& =-e^{-i \phi} \sin \frac{\theta}{2}|K\rangle^{(0)}+\cos \frac{\theta}{2}\left|K^{\prime}\right\rangle^{(0)},
\end{aligned}
$$

in terms of the phases $\theta$ and $\phi$, allowing for visualizing the valley-polarized exciton states as pseudospin in the
Bloch sphere as presented in Fig. 3. Note that the inplane angle given by $\phi=-\arg \left[\Delta_{K K^{\prime}}\right]$ is associated with the valley-mixing $e-h$ exchange interaction and the polar angle $\theta=\cos ^{-1}\left[\frac{\Delta \widetilde{E}_{K, K^{\prime}}^{X}}{\sqrt{\left(\Delta \widetilde{E}_{K, K^{\prime}}^{X}\right)^{2}+4\left|\widetilde{\Delta}_{K K^{\prime}}\right|^{2}}}\right]$, where $\Delta \widetilde{E}_{K, K^{\prime}}^{X}=E_{K}^{X(0)}-$ $E_{K^{\prime}}^{X(0)}+\widetilde{\Delta}_{K K}-\widetilde{\Delta}_{K^{\prime} K^{\prime}}$ is made by the energy difference between the valley-uncoupled exciton basis states. Figure 3 presents the valley pseudospin texture of finite-momentum exciton states over the BZ in the exciton-momentum space. The upward (downward) orientation of pseudospin with $\theta \rightarrow 0(\pi)$ represents the full $K\left(K^{\prime}\right)$-valley polarization, e.g., the MFDX exciton states in the $K_{\mathrm{ex}}\left(K_{\mathrm{ex}}^{\prime}\right)$ valley. By contrast, the in-plane orientation of valley pseudospin with $\theta \sim \pi / 2$ indicates the complete intermixing of $K$ and $K^{\prime}$ valleys such as the valleydepolarized exciton states lying around the $\Gamma_{\mathrm{ex}}$ and $M_{\mathrm{ex}}$ points. The intriguing pseudospin texture of the finite-momentum exciton over the $\mathrm{BZ}$ is in agreement with the prediction of the symmetry analysis presented in Sec. II B.

\section{B. Indirect phonon-assisted luminescences from the finite-momentum exciton}

Despite the violation of the momentum selection rules, those highly valley-polarized MFDXs, e.g., the $K^{\prime} K$ intervalley MFDX states $|D\rangle$, can be luminescent by means of the indirect radiative transitions, virtually via $\mathrm{BX}$ states $|B\rangle$ with 


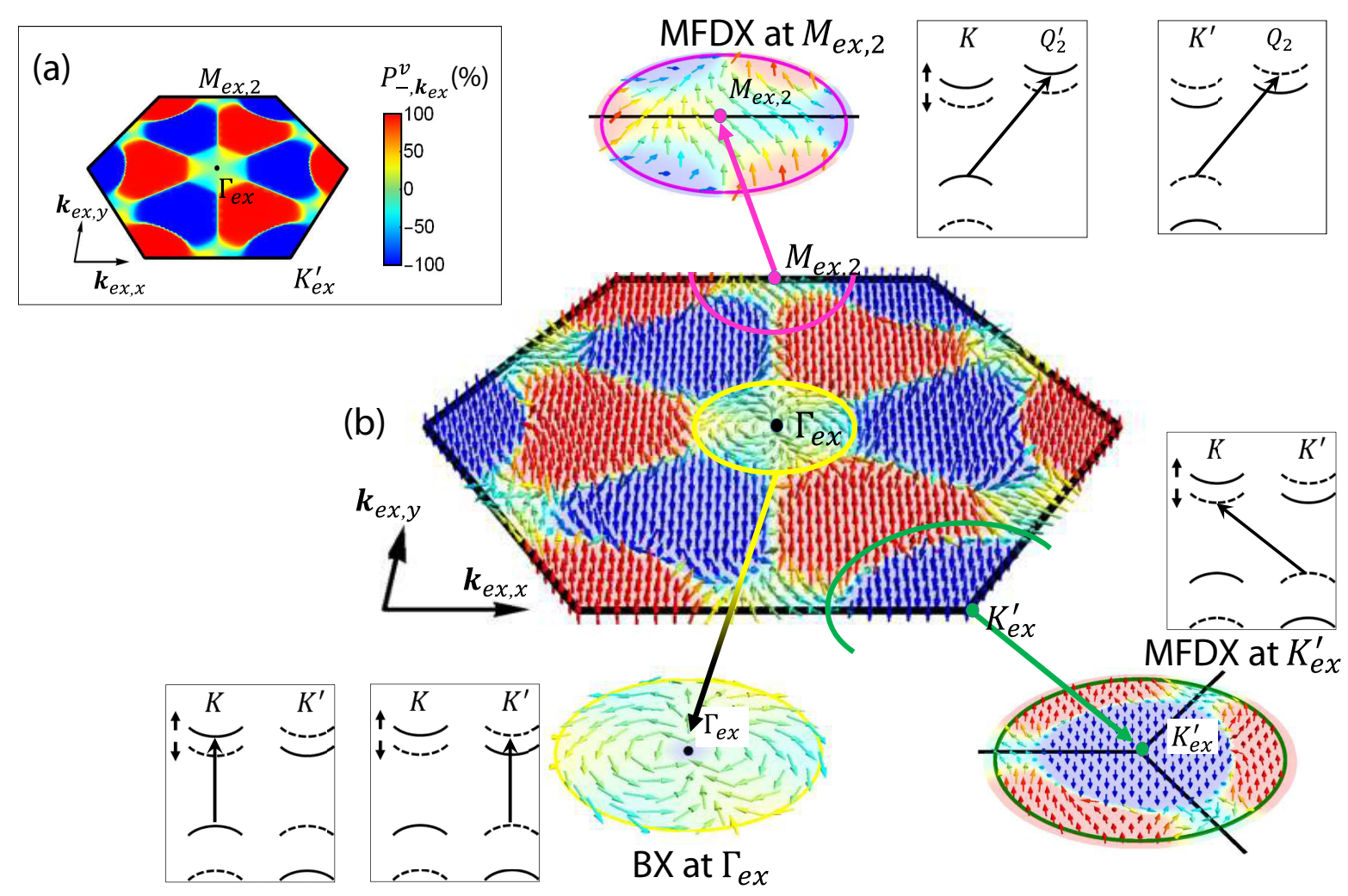

FIG. 3. (a) Distribution of the degree of valley polarizations of the lowest exciton band states $\left|S=-, \boldsymbol{k}_{\mathrm{ex}}\right\rangle$, over the first BZ, which is duplicated from Fig. 2(f) for the reference of (b). (b) The valley pseudospin texture over the BZ visualizes the momentum-dependent valley polarizations of finite-momentum exciton states. The upward (downward) orientation of pseudospin in red (blue) color represents the $K\left(K^{\prime}\right)$-valley polarization. For example, the MFDX exciton states in the $K_{\mathrm{ex}}^{\prime}$ valley are highly $K^{\prime}$-valley polarized and visualized by the downward pseudospin in blue color. By contrast, the BX states around the $\Gamma_{\mathrm{ex}}$ point as well as the MFDX exciton states around $M_{\mathrm{ex}, i}$ points are valley depolarized and visualized by the in-plane-oriented pseudospin (green colored arrows).

the assistance of phonon emission or absorption [see Fig. 4(a) for illustration]. After the reveal of symmetry-dictated valley properties of MFDXs, our next concern is how much the superior valley polarizations of the MFDXs can be really converted to the optical polarization as the intermediate BX states are severely subjected to the EHEI in the indirect transitions.

\section{Pronounced indirect luminescence at cryogenic temperature}

For the analysis of phonon-assisted transition, we consider the extended exciton-photon-phonon system with the Hamiltonian $\hat{H}=\hat{H}_{X}+\hat{H}_{v}+\hat{H}_{\mathrm{ph}}+\hat{H}_{X-v}+\hat{H}_{X-\mathrm{ph}}$, where $\hat{H}_{X}=$ $\sum_{S \boldsymbol{k}_{\mathrm{ex}}} E_{S, \boldsymbol{k}_{\mathrm{e}}}^{X} \hat{X}_{S, \boldsymbol{k}_{\mathrm{e}}}^{\dagger} \hat{X}_{S, \boldsymbol{k}_{\mathrm{ex}}}$ stands for the single-exciton Hamiltonian, $\hat{X}\left(\hat{X}^{\dagger}\right)$ is the operator annihilating (creating) an exciton, $\hat{H}_{v}=\sum_{\epsilon} \sum_{\boldsymbol{k}_{v}} \hbar \omega_{\boldsymbol{k}_{v}}^{\epsilon} \hat{a}_{\boldsymbol{\epsilon}, \boldsymbol{k}_{v}}^{\dagger} \hat{a}_{\boldsymbol{\epsilon}, \boldsymbol{k}_{v}}\left(\hat{H}_{\mathrm{ph}}=\sum_{\lambda} \sum_{q} \hbar \Omega_{q}^{\lambda} \hat{b}_{\lambda, q}^{\dagger} \hat{b}_{\lambda, \boldsymbol{q}}\right)$ is the Hamiltonian of photon (phonon) reservoir, $\omega_{\boldsymbol{k}_{v}}^{\epsilon}$ is the frequency of the $\boldsymbol{\epsilon}$-polarized photon with the wave vector $\boldsymbol{k}_{v}$, $\Omega_{q}^{\lambda}$ is the frequency of the $\lambda$-kind phonon with the wave vector $\boldsymbol{q}[60]$, and $\hat{a} / \hat{a}^{\dagger}\left(\hat{b} / \hat{b}^{\dagger}\right)$ are the particle operators that annihilate/create a photon (phonon). $\hat{H}_{X-v}\left(\hat{H}_{X-\mathrm{ph}}\right)$ represents the Hamiltonian of exciton-photon (exciton-phonon) interaction in terms of the $\boldsymbol{\epsilon}(\lambda)$-dependent coupling constants $\eta_{S, \boldsymbol{k}_{\mathrm{ex}}}^{\boldsymbol{\epsilon}, \boldsymbol{k}_{\mathrm{v}}}\left(g_{S^{\prime}, \boldsymbol{k}_{\mathrm{ex}}^{\prime}: S, \boldsymbol{k}_{\mathrm{ex}}}^{\lambda, \boldsymbol{q}}\right)$ (see Ref. [43] for the explicit expressions). The effects of the presence of MFDXs on the direct PL of TMD-MLs have been investigated by Ref. [13]. Here our main attention is on the indirect phonon-assisted transitions from the lowest spinlike $K K^{\prime} / K^{\prime} K$ MFDX states in the $K_{\mathrm{ex}} / K_{\mathrm{ex}}^{\prime}$ valleys whose superiorly high polarizations were recently identified by experiments $[16,18]$. Though in reality PL processes essentially involve also the complex dynamics of exciton relaxation [13], here, for the pure purpose to assess the valley-to-optical polarization conversion, the analysis below is focused on the intrinsic polarization properties of indirect transitions alone.

In a phonon-assisted radiative transition from a $\mathrm{WSe}_{2}-$ ML, an exciton initially in the lowest intervalley MFDX state $\left|S=-, \boldsymbol{k}_{\mathrm{ex}}=\boldsymbol{K}_{\mathrm{ex}} / \boldsymbol{K}_{\mathrm{ex}}^{\prime}\right\rangle \equiv|D\rangle$ is virtually transferred to the intermediate BX states, $\left|S^{\prime}, \boldsymbol{k}_{\mathrm{ex}}^{\prime} \in L . C.\right\rangle \equiv\left|S^{\prime}, B\right\rangle$, assisted by the phonon with $\boldsymbol{k}_{\mathrm{ex}}-\boldsymbol{k}_{\mathrm{ex}}^{\prime} \equiv \boldsymbol{q}_{0}$ and then, from $\left|S^{\prime}, B\right\rangle$, spontaneously emits a photon of wave vector $\boldsymbol{k}_{v}$ whose in-plane projection matches the $2 \mathrm{D}$-exciton wave vector $\boldsymbol{k}_{v, \|}=\boldsymbol{k}_{\mathrm{ex}}^{\prime}$ [see Fig. 4(b) for schematic illustration]. In Ref. [43] we derive the rates of the phonon-assisted radiative transitions from a MFDX state $|D\rangle$ of $\mathrm{WSe}_{2}-\mathrm{ML}$, as formulated by $\Gamma_{D}^{(2) \lambda, \pm}=\bar{\gamma}_{D}^{(2) \lambda, \pm} \delta\left(E_{D}^{X} \pm \hbar \Omega_{\mp \boldsymbol{q}_{0}}^{\lambda}-\hbar \omega_{\boldsymbol{k}_{v}}\right)$, where $\lambda$ labels the kind of the involved phonons, $+/-$ indicates the phonon absorption/emission, $\boldsymbol{q}_{0}\left(\boldsymbol{k}_{v}\right)$ is the wave vector of involved phonon (emitted photon), and $\bar{\gamma}_{D}^{(2) \lambda, \pm}$ is implicitly defined to measure the indirect transition rate under the condition of energy conservation. As a generalized theory, we 
(a)

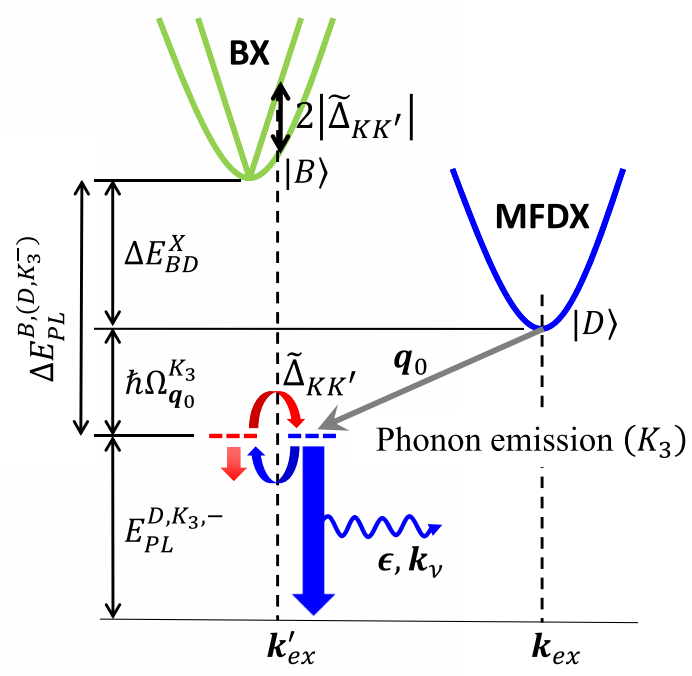

(b)
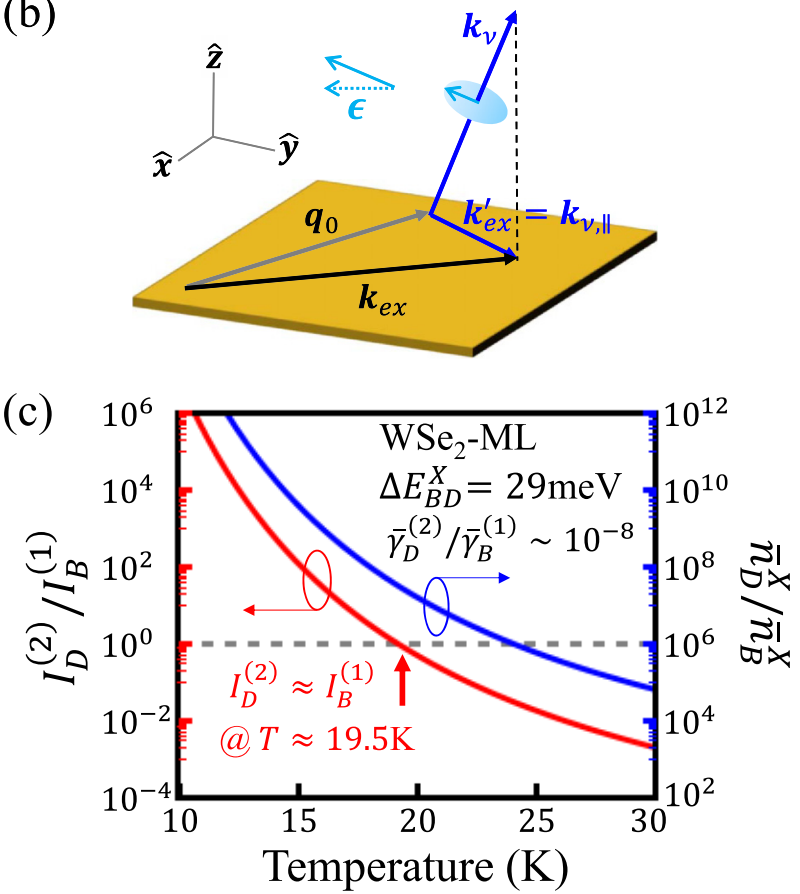

FIG. 4. (a) Illustrative schematics of an indirect phonon-assisted radiative transition from a MFDX state $|D\rangle$ and mediated by a BX state $|B\rangle$. The initial MFDX state $|D\rangle$ virtually transfers to the intermediate BXs $| \pm, B\rangle$ by emitting a phonon with momentum $\boldsymbol{q}_{0}=\boldsymbol{k}_{\mathrm{ex}}-\boldsymbol{k}_{\mathrm{ex}}^{\prime}$, and then emits a photon carrying momentum $\boldsymbol{k}_{v}$. (b) The wave vectors of the involved exciton, phonon and photon under the law of momentum conservation. (c) Blue curve: The ratio of the thermal population in $|D\rangle$ and that in $|B\rangle$ as a function of temperature $T$. Red curve: The intensity ratio of the second-order indirect transition from $|D\rangle$ and the direct one from $|B\rangle$.

consider the both emission and absorption of the all phonon modes ( $K_{1}$ and $K_{3}$ modes) allowed by the selection rules given by Refs. $[18,61]$ for the indirect transitions and numerically calculate the rates of the phonon-assisted transitions taking the DFT-based parameters of carrier-phonon interactions for $\mathrm{WSe}_{2}$-MLs (see Ref. [43] for details) [60,62]. From the numerical results of Fig. S5 in Ref. [43], the dominant phonon-assisted channel for the indirect transitions from the $K^{\prime} K$ MFDX states is via the emission of $K_{3}$-phonon modes $\left(\bar{\gamma}_{D}^{(2) K_{3},-}\right)$, consistent with the experimental identifications [16-20,30,61].

Analytically [43], the ratio of the indirect $K_{3}$-phononassisted radiative transition from a MFDX state and the direct radiative transition from a $\mathrm{BX}$ state is derived as $\bar{\gamma}_{D}^{(2)} / \bar{\gamma}_{B}^{(1)}=$ $\pi\left(\frac{a_{0}}{\lambda}\right)^{2}\left|\frac{\hbar g_{B D} \sqrt{N}}{\Delta E_{B D}^{X}+\hbar \Omega_{q_{0}}^{K_{0}}}\right|^{2}$, where $a_{0}=0.3316 \mathrm{~nm}$ is the lattice constant of $\mathrm{WSe}_{2}$-ML [36], $\lambda \approx 750 \mathrm{~nm}$ is the wavelength of the emitted light from $1 s$ exciton, $N$ is the total number of primitive cells of the material, $\hbar g_{B D}=\frac{12.3}{\sqrt{N}} \mathrm{meV}$ is evaluated as the effective exciton-phonon coupling between $|D\rangle$ and $|B\rangle[43,60], \Delta E_{B D}^{X} \equiv E_{B}^{X}-E_{D}^{X} \approx 29 \mathrm{meV}$, and $\hbar \Omega_{q_{0}}^{K_{3}} \approx$ $26 \mathrm{meV}$ [18]. With the use of those parameters, we count $\bar{\gamma}_{D}^{(2)} / \bar{\gamma}_{B}^{(1)} \sim 10^{-8}$, showing the extremely low rate of the phonon-assisted radiative transition from a MFDX.

The transition rate of indirect PL is very low since only a very small portion of intermediate exciton states in the $\boldsymbol{k}_{\mathrm{ex}}$ Brillouin zone that are optically active, which is measured by the area ratio of the light cone and the entire zone, i.e., $\left(\frac{a_{0}}{\lambda}\right)^{2}=\left(\frac{k_{c}}{|\boldsymbol{G}|}\right)^{2}$, where $|\boldsymbol{G}|=\frac{2 \pi}{a_{0}}$ and $k_{c}=\frac{2 \pi}{\lambda}$. Nevertheless, the intensity of photon emission from a radiative transition is determined by the product of the bare transition rate and the averaged number of exciton in the initial state, i.e., $I_{B / D}^{(1) /(2)} \propto$ $\bar{\gamma}_{B / D}^{(1) /(2)} \bar{n}_{B / D}^{X}(T)$, where $\bar{n}_{B / D}^{X}(T) \propto e^{-E_{B / D}^{X} / k_{B} T}$ is in the Boltzmann statistics. For WSe $\mathrm{WL}_{2}$-ML, the lowest $K_{\mathrm{ex}} / K_{\mathrm{ex}}^{\prime} \mathrm{MFDX}$ states are so much lower than the BX ones and host the tremendously high exciton population at low temperature. Figure $4(\mathrm{c})$ shows $\bar{n}_{D}^{X} / \bar{n}_{B}^{X} \gtrsim 10^{8}$ and $I_{D}^{(2)} / I_{B}^{(1)}>1$ at low temperatures $T<19.5 \mathrm{~K}$ for a $\mathrm{WSe}_{2}$-ML. This accounts for the recently observed high intensity of the $K_{3}$-phonon-assisted PLs from $\mathrm{WSe}_{2}$-MLs of Refs. [16-19,30,63], as summarized in Ref. [43].

\section{Superior valley-to-optical polarization conversion}

At last, let us examine the optical polarization $P_{D}^{o(2)} \equiv \frac{I_{D}^{(2)}\left(\boldsymbol{\epsilon}_{+}\right)-I_{D}^{(2)}\left(\boldsymbol{\epsilon}_{-}\right)}{I_{D}^{(2)}\left(\boldsymbol{\epsilon}_{+}\right)+I_{D}^{(2)}\left(\boldsymbol{\epsilon}_{-}\right)} \quad$ of the phonon-assisted transition from the valley-mixed MFDX state $|D\rangle=\alpha_{D}^{K}\left|K, \boldsymbol{k}_{\mathrm{ex}}\right\rangle^{(0)}+$ $\alpha_{D}^{K^{\prime}}\left|K^{\prime}, \boldsymbol{k}_{\text {ex }}\right\rangle^{(0)}$, where $\boldsymbol{\epsilon}_{ \pm} \equiv \frac{1}{\sqrt{2}}(\hat{\boldsymbol{x}} \pm i \hat{\boldsymbol{y}})$ is the circular polarization vector. As detailed in [43], we show that the optical polarization of an indirect phonon-assisted transition via the $\mathrm{BX}$ intermediate states $P_{D}^{o(2)}$ can be related to the valley polarization of the initial MFDX state $P_{D}^{v}$ by

$$
P_{D}^{o(2)} \approx P_{D}^{v}\left\{1-4 \operatorname{Re}\left[\widetilde{\beta}_{B D}^{K K^{\prime}}\left(\boldsymbol{k}_{v, \|}\right)\left(\alpha_{D}^{K}\right)^{*} \alpha_{D}^{K^{\prime}}\right]\right\},
$$

where $\widetilde{\beta}_{B D}^{K K^{\prime}} \equiv-\frac{\widetilde{\Delta}_{K K^{\prime}}\left(\boldsymbol{k}_{v, \|}\right)}{\Delta E_{B D}^{X}+\hbar \Omega_{q_{0}}^{K_{3}}}$ arises from the EHEI $\widetilde{\Delta}_{K K^{\prime}}$ in the intermediate $\mathrm{BX}$ states through which the $K\left(K^{\prime}\right)$-valley component in $|D\rangle$ is cross converted and the optical polarization could be degraded. However, the small value of $\left|\widetilde{\beta}_{B D}^{K K^{\prime}}\left(\boldsymbol{k}_{v, \|}\right)\right| \sim$ 
$10^{-2}$ estimated by taking $\left|\widetilde{\Delta}_{K K^{\prime}}\right| \sim 1 \mathrm{meV}$ and $\left(\Delta E_{B D}^{X}+\right.$ $\left.\hbar \Omega_{q_{0}}^{K_{3}}\right) \approx 55 \mathrm{meV}$ indicates that $P_{D}^{o(2)} \approx P_{D}^{v}$. This reveals the native suppression of exchange-induced depolarization in the second-order PL process and accounts for the recently observed high polarizations of the indirect phonon-assisted PLs from $\mathrm{WSe}_{2}$-MLs (see Table S5 in Ref. [43]) [16,18],

\section{CONCLUSION}

In conclusion, we present a comprehensive theoretical investigation of the full-zone landscape of the valley-polarized MFDX states of $\mathrm{WSe}_{2}$-MLs. We show the guiding principle for the prediction of the momentum-dependent valley polarizations of MFDX states under the enforcement of crystal symmetry. Specifically, the valley polarizations of the MFDX states lying in the $K_{\mathrm{ex}}, K_{\mathrm{ex}}^{\prime}$, and $Q_{\mathrm{ex}, i}$ valleys (along the
$\overline{\Gamma_{\mathrm{ex}} M_{\mathrm{ex}, i}}$ paths) in the exciton-momentum space are realized inherently deterministic to be high (nearly vanishing). More importantly, we show the high polarizations of the intervalley MFDX states are fully transferable to the optical polarization in the indirect phonon-assisted radiative transitions, shedding light on the prospect of those long-lived, optically accessible and highly valley-polarized MFDXs for realizing the valleybased photonics.

\section{ACKNOWLEDGMENTS}

P.-Y.L. and S.-J.C. thank M. Bieniek and P. Hawrylak for fruitful discussion. This study is supported by the Ministry of Science and Technology (Taiwan), under Contracts MOST 109-2639-E-009-001 and 109-2112-M-009-018-MY3, and by National Center for High-Performance Computing (NCHC), Taiwan.
[1] K. F. Mak, C. Lee, J. Hone, J. Shan, and T. F. Heinz, Atomically Thin $\mathrm{MoS}_{2}$ : A New Direct-Gap Semiconductor, Phys. Rev. Lett. 105, 136805 (2010)

[2] D. Xiao, G.-B. Liu, W. Feng, X. Xu, and W. Yao, Coupled Spin and Valley Physics in Monolayers of $\mathrm{MoS}_{2}$ and Other Group-VI Dichalcogenides, Phys. Rev. Lett. 108, 196802 (2012).

[3] X. Xu, W. Yao, D. Xiao, and T. F. Heinz, Spin and pesupospins in layered transition metal dichalcogenides, Nat. Phys. 10, 343 (2014).

[4] H. Yu, G.-B. Liu, P. Gong, X. Xu, and W. Yao, Dirac cones and Dirac saddle points of bright excitons in monolayer transition metal dichalcogenides, Nat. Commun. 5, 3876 (2014).

[5] H. Zeng, J. Dai, W. Yao, D. Xiao, and X. Cui, Valley polarization in $\mathrm{MoS}_{2}$ monolayers by optical pumping, Nat. Nanotechnol. 7, 490 (2012).

[6] K. F. Mak, D. Xiao, and J. Shan, Light-valley interactions in 2D semiconductors, Nat. Photonics 12, 451 (2018).

[7] G. Wang, A. Chernikov, M. M. Glazov, T. F. Heinz, X. Marie, T. Amand, and B. Urbaszek, Colloquium: Excitons in atomically thin transition metal dichalcogenides, Rev. Mod. Phys. 90, 021001 (2018).

[8] T. Smoleński, M. Goryca, M. Koperski, C. Faugeras, T. Kazimierczuk, A. Bogucki, K. Nogajewski, P. Kossacki, and M. Potemski, Tuning Valley Polarization in a $\mathrm{WSe}_{2}$ Monolayer with a Tiny Magnetic Field, Phys. Rev. X 6, 021024 (2016).

[9] T. Yu and M. W. Wu, Valley depolarization due to intervalley and intravalley electron-hole exchange interactions in monolayer $\mathrm{MoS}_{2}$, Phys. Rev. B 89, 205303 (2014).

[10] K. Hao, G. Moody, F. Wu, C. K. Dass, L. Xu, C.-H. Chen, L. Sun, M.-Y. Li, L.-J. Li, A. H. MacDonald, and X. Li, Direct measurement of exciton valley coherence in monolayer $\mathrm{WSe}_{2}$, Nat. Phys. 12, 677 (2016).

[11] G. Wang, L. Bouet, D. Lagarde, M. Vidal, A. Balocchi, T. Amand, X. Marie, and B. Urbaszek, Valley dynamics probed through charged and neutral exciton emission in monolayer $\mathrm{WSe}_{2}$, Phys. Rev. B 90, 075413 (2014).
[12] M. Glazov, E. Ivchenko, G. Wang, T. Amand, X. Marie, B. Urbaszek, and B. Liu, Spin and valley dynamics of excitons in transition metal dichalcogenide monolayers, Phys. Status Solidi (b) 252, 2349 (2015).

[13] M. Selig, F. Katsch, S. Brem, G. F. Mkrtchian, E. Malic, and A. Knorr, Suppression of intervalley exchange coupling in the presence of momentum-dark states in transition metal dichalcogenides, Phys. Rev. Res. 2, 023322 (2020).

[14] D. Y. Qiu, T. Cao, and S. G. Louie, Nonanalyticity, Valley Quantum Phases, and Lightlike Exciton Dispersion in Monolayer Transition Metal Dichalcogenides: Theory and First-Principles Calculations, Phys. Rev. Lett. 115, 176801 (2015).

[15] K. B. Simbulan, T.-D. Huang, G.-H. Peng, F. Li, O. J. Gomez Sanchez, J.-D. Lin, C.-I. Lu, C.-S. Yang, J. Qi, S.-J. Cheng, T.-H. Lu, and Y.-W. Lan, Selective photoexcitation of finitemomentum excitons in monolayer $\mathrm{MoS}_{2}$ by twisted light, ACS Nano 15, 3481 (2021).

[16] Z. Li, T. Wang, C. Jin, Z. Lu, Z. Lian, Y. Meng, M. Blei, M. Gao, T. Taniguchi, K. Watanabe, T. Ren, T. Cao, S. Tongay, D. Smirnov, L. Zhang, and S.-F. Shi, Momentum-dark intervalley exciton in monolayer tungsten diselenide brightened via chiral phonon, ACS Nano 13, 14107 (2019).

[17] E. Liu, J. van Baren, C.-T. Liang, T. Taniguchi, K. Watanabe, N. M. Gabor, Y.-C. Chang, and C. H. Lui, Multipath Optical Recombination of Intervalley Dark Excitons and Trions in Monolayer $\mathrm{WSe}_{2}$, Phys. Rev. Lett. 124, 196802 (2020).

[18] M. He, P. Rivera, D. V. Tuan, N. P. Wilson, M. Yang, T Taniguchi, K. Watanabe, J. Yan, D. G. Mandrus, H. Yu, H. Dery, W. Yao, and $\mathrm{X}$. Xu, Valley phonons and exciton complexes in a monolayer semiconductor, Nat. Commun. 11, 618 (2020).

[19] S. Brem, A. Ekman, D. Christiansen, F. Katsch, M. Selig, C. Robert, X. Marie, B. Urbaszek, A. Knorr, and E. Malic, Phonon-assisted photoluminescence from indirect excitons in monolayers of transition-metal dichalcogenides, Nano Lett. 20 , 2849 (2020).

[20] E. Liu, J. van Baren, T. Taniguchi, K. Watanabe, Y.-C. Chang, and C. H. Lui, Valley-selective chiral phonon replicas of dark 
excitons and trions in monolayer $\mathrm{WSe}_{2}$, Phys. Rev. Res. 1, 032007(R) (2019).

[21] D. Bao, A. G. del Águila, T. T. H. Do, S. Liu, J. Pei, and Q. Xiong, Probing momentum-indirect excitons by near-resonance photoluminescence excitation spectroscopy in $\mathrm{WS}_{2}$ monolayer, 2D Mater. 7, 031002 (2020).

[22] L. Wu, Y. Chen, H. Zhou, and H. Zhu, Ultrafast energy transfer of both bright and dark excitons in $2 \mathrm{~d}$ van der Waals heterostructures beyond dipolar coupling, ACS Nano 13, 2341 (2019).

[23] A. Koitzsch, A.-S. Pawlik, C. Habenicht, T. Klaproth, R. Schuster, B. Büchner, and M. Knupfer, Nonlocal dielectric function and nested dark excitons in $\mathrm{MoS}_{2}$, npj 2D Mater. Appl. 3, 41 (2019).

[24] J. Hong, R. Senga, T. Pichler, and K. Suenaga, Probing Exciton Dispersions of Freestanding Monolayer $\mathrm{WSe}_{2}$ by MomentumResolved Electron Energy-Loss Spectroscopy, Phys. Rev. Lett. 124, 087401 (2020).

[25] M. Feierabend, S. Brem, A. Ekman, and E. Malic, Brightening of spin- and momentum-dark excitons in transition metal dichalcogenides, 2D Mater. 8, 015013 (2021).

[26] X. Jiang, Q. Zheng, Z. Lan, W. A. Saidi, X. Ren, and J. Zhao, Real-time GW-BSE investigations on spin-valley exciton dynamics in monolayer transition metal dichalcogenide, Sci. Adv. 7, 3759 (2021).

[27] R. Wallauer, R. Perea-Causin, L. Münster, S. Zajusch, S. Brem, J. Güdde, K. Tanimura, K.-Q. Lin, R. Huber, E. Malic, and U. Höfer, Direct observation of ultrafast dark exciton formation in monolayer $\mathrm{WS}_{2}$, Nano Lett. 21, 5867 (2021).

[28] J. Madéo, M. K. L. Man, C. Sahoo, M. Campbell, V. Pareek, E. L. Wong, A. Al-Mahboob, N. S. Chan, A. Karmakar, M. B. M. Krishna, X. Li, T. F. Heinz, T. Cao, and K. M. Dani, Directly visualizing the momentum-forbidden dark excitons and their dynamics in atomically thin semiconductors, Science 370, 1199 (2020).

[29] C. Middleton, Photoelectrons shine a light on dark excitons, Phys. Today 74(2), 16 (2021).

[30] S.-Y. Chen, M. Pieczarka, M. Wurdack, E. Estrecho, T. Taniguchi, K. Watanabe, J. Yan, E. A. Ostrovskaya, and M. S. Fuhrern, Long-lived populations of momentum- and spinindirect excitons in monolayer $\mathrm{WSe}_{2}$, arXiv:2009.09602.

[31] Z. Wang, D. A. Rhodes, K. Watanabe, T. Taniguchi, J. C. Hone, J. Shan, and K. F. Mak, Evidence of high-temperature exciton condensation in two-dimensional atomic doulbe layers, Nature (London) 574, 76 (2019).

[32] Y. Mazuz-Harpaz, K. Cohen, M. Leveson, K. West, L. Pfeiffer, M. Khodas, and R. Rapaport, Dynamical formation of a strongly correlated dark condensate of dipolar excitons, Proc. Natl. Acad. Sci. USA 116, 18328 (2019).

[33] L. Sigl, F. Sigger, F. Kronowetter, J. Kiemle, J. Klein, K. Watanabe, T. Taniguchi, J. J. Finley, U. Wurstbauer, and A. W. Holleitner, Signatures of a degenerate many-body state of interlayer excitons in a van der Waals heterostack, Phys. Rev. Res. 2, 042044(R) (2020).

[34] M. Combescot, R. Combescot, and F. Dubin, Bose-Einstein condensation and indirect excitons: A review, Rep. Prog. Phys. 80, 066501 (2017).

[35] T. Deilmann and K. S. Thygesen, Finite-momentum exciton landscape in mono-and bilayer transition metal dichalcogenides, 2D Mater. 6, 035003 (2019).
[36] G.-H. Peng, P.-Y. Lo, W.-H. Li, Y.-C. Huang, Y.-H. Chen, C.-H. Lee, C.-K. Yang, and S.-J. Cheng, Distinctive signatures of the spin- and momentum-forbidden dark exciton states in the photoluminescence of strained $\mathrm{WSe}_{2}$ monolayers under thermalization, Nano Lett. 19, 2299 (2019).

[37] L. J. Sham and T. M. Rice, Many-particle derivation of the effective-mass equation for the Wannier exciton, Phys. Rev. 144, 708 (1966).

[38] W. Hanke and L. J. Sham, Many-particle effects in the optical spectrum of a semiconductor, Phys. Rev. B 21, 4656 (1980).

[39] M. Rohlfing and S. G. Louie, Electron-Hole Excitations in Semiconductors and Insulators, Phys. Rev. Lett. 81, 2312 (1998).

[40] F. Wu, F. Qu, and A. H. MacDonald, Exciton band structure of monolayer $\mathrm{MoS}_{2}$, Phys. Rev. B 91, 075310 (2015).

[41] M. Bieniek, L. Szulakowska, and P. Hawrylak, Band nesting and exciton spectrum in monolayer $\mathrm{MoS}_{2}$, Phys. Rev. B 101, 125423 (2020).

[42] R. Vasconcelos, H. Bragança, F. Qu, and J. Fu, Dark exciton brightening and its engaged valley dynamics in monolayer $\mathrm{WSe}_{2}$, Phys. Rev. B 98, 195302 (2018).

[43] See Supplemental Material at http://link.aps.org/supplemental/ 10.1103/PhysRevResearch.3.043198, which includes the detailed information about the first-principles computation, symmetry analysis, the matrix elements of $e-h$ direct and exchange Coulomb interactions, pseudospin exciton model, and the theory for the optical polarizations of phonon-assisted indirect photoluminescences.

[44] L. V. Keldysh, Coulomb interaction in thin semiconductor and semimetal films, Pis'ma Zh. Eksp. Teor. Fiz. 29, 716 (1979) [JETP Lett. 29, 658 (1979)].

[45] P. Cudazzo, I. V. Tokatly, and A. Rubio, Dielectric screening in two-dimensional insulators: Implications for excitonic and impurity states in graphane, Phys. Rev. B 84, 085406 (2011).

[46] T. C. Berkelbach, M. S. Hybertsen, and D. R. Reichman, Theory of neutral and charged excitons in monolayer transition metal dichalcogenides, Phys. Rev. B 88, 045318 (2013).

[47] A. V. Stier, N. P. Wilson, K. A. Velizhanin, J. Kono, X. Xu, and S. A. Crooker, Magnetooptics of Exciton Rydberg States in a Monolayer Semiconductor, Phys. Rev. Lett. 120, 057405 (2018).

[48] E. Ridolfi, C. H. Lewenkopf, and V. M. Pereira, Excitonic structure of the optical conductivity in $\mathrm{MoS}_{2}$ monolayers, Phys. Rev. B 97, 205409 (2018).

[49] M. L. Trolle, T. G. Pedersen, and V. Véniard, Model dielectric function for 2D semiconductors including substrate screening, Sci. Rep. 7, 39844 (2017).

[50] G. Kresse and J. Furthmüller, Efficient iterative schemes for $a b$ initio total-energy calculations using a plane-wave basis set, Phys. Rev. B 54, 11169 (1996).

[51] A. V. Krukau, O. A. Vydrov, A. F. Izmaylov, and G. E. Scuseria, Influence of the exchange screening parameter on the performance of screened hybrid functionals, J. Chem. Phys. 125, 224106 (2006).

[52] K. Kośmider, J. W. González, and J. Fernández-Rossier, Large spin splitting in the conduction band of transition metal dichalcogenide monolayers, Phys. Rev. B 88, 245436 (2013).

[53] B. Scharf, T. Frank, M. Gmitra, J. Fabian, I. Žutić, and V. Perebeinos, Excitonic stark effect in $\mathrm{MoS}_{2}$ monolayers, Phys. Rev. B 94, 245434 (2016). 
[54] J. L. Lado and J. Fernández-Rossier, Landau levels in 2D materials using Wannier Hamiltonians obtained by first principles, 2D Mater. 3, 035023 (2016).

[55] A. A. Mostofi, J. R. Yates, Y.-S. Lee, I. Souza, D. Vanderbilt, and N. Marzari, Wannier90: A tool for obtaining maximallylocalised Wannier functions, Comput. Phys. Commun. 178, 685 (2008).

[56] A. A. Mostofi, J. R. Yates, G. Pizzi, Y.-S. Lee, I. Souza, D. Vanderbilt, and N. Marzari, An updated version of Wannier90: A tool for obtaining maximally-localised Wannier functions, Comput. Phys. Commun. 185, 2309 (2014).

[57] D. Y. Qiu, F. H. da Jornada, and S. G. Louie, Optical Spectrum of $\mathrm{MoS}_{2}$ : Many-Body Effects and Diversity of Exciton States, Phys. Rev. Lett. 111, 216805 (2013).

[58] A. Chernikov, T. C. Berkelbach, H. M. Hill, A. Rigosi, Y. Li, O. B. Aslan, D. R. Reichman, M. S. Hybertsen, and T. F. Heinz, Exciton Binding Energy and Nonhydrogenic Rydberg Series in Monolayer $\mathrm{WS}_{2}$, Phys. Rev. Lett. 113, 076802 (2014).
[59] C. Robert, T. Amand, F. Cadiz, D. Lagarde, E. Courtade, M. Manca, T. Taniguchi, K. Watanabe, B. Urbaszek, and X. Marie, Fine structure and lifetime of dark excitons in transition metal dichalcogenide monolayers, Phys. Rev. B 96, 155423 (2017).

[60] Z. Jin, X. Li, J. T. Mullen, and K. W. Kim, Intrinsic transport properties of electrons and holes in monolayer transition-metal dichalcogenides, Phys. Rev. B 90, 045422 (2014).

[61] H. Zhu, J. Yi, M.-Y. Li, J. Xiao, L. Zhang, C.-W. Yang, R. A. Kaindl, L.-J. Li, Y. Wang, and X. Zhang, Observation of chiral phonons, Science 359, 579 (2018).

[62] K. Kaasbjerg, K. S. Thygesen, and K. W. Jacobsen, Phononlimited mobility in $n$-type single-layer $\mathrm{MoS}_{2}$ from first principles, Phys. Rev. B 85, 115317 (2012).

[63] Z. Ye, L. Waldecker, E. Y. Ma, D. Rhodes, A. Antony, B. Kin, X.-X. Zhang, M. Deng, Y. Jiang, Z. Lu, D. Smirnov, K. Watanabe, T. Taniguchi, J. Hone, and T. F. Heinz, Efficient generation of neutral and charged biexcitons in encapsulated $\mathrm{WSe}_{2}$ monolayers, Nat. Commun. 9, 3718 (2018). 